Available online at http://jnfa.mathres.org
MATHRES Journal of Nonlinear Functional Analysis
Ants

\title{
A FRACTIONAL DIFFERENTIAL EQUATION MODEL FOR BIKE SHARE SYSTEMS
}

\author{
JOHN R. GRAEF ${ }^{1, *}$, SHEN-SHYANG HO ${ }^{2}$, LINGJU KONG $^{1}$, MIN WANG $^{3}$ \\ ${ }^{1}$ Department of Mathematics, University of Tennessee at Chattanooga, Chattanooga, TN 37403, USA \\ ${ }^{2}$ Department of Computer Science, Rowan University, Glassboro, NJ 08028, USA \\ ${ }^{3}$ Department of Mathematics, Kennesaw State University, Marietta, GA 30060, USA
}

\begin{abstract}
In this paper, a fractional differential equation model is developed to describe the bike share station status based on data analysis of historical data of bike share systems in Philadelphia and Atlanta. The analytic solution and a related control problem are investigated as well.
\end{abstract}

Keywords. Stochastic differential equation; Bike share systems; Fractional differential equation models.

2010 Mathematics Subject Classification. 34A08, 93B05.

\section{INTRODUCTION}

Bike share systems have become more and more popular around the world. The reader is referred to [1] and references therein for a brief review of bike share history. In the USA, the usage of bike share systems has soared from 320,000 to $35 \mathrm{M}$ in 8 years [2, 3]; Fig. 1 shows a historical growth chart of bike share systems in the USA. One major user complaint about bike share systems around the world is the occurrence of a bike station being completely empty or full. This has been a big issue that has impacted the usage of bike share systems. For instance, a big portion of bike share system users regularly rely on them as their commute solution during rush hours (7-9 am and 4-7 pm) [3]. The shortage of docks (full station) or bikes (empty station) may prolong their commute time and force them to seek alternative commute solutions. This will potentially decrease the usage of bikes in the future. Therefore, the rebalancing or redistribution of bikes among bike stations is needed to supplement the bikes or free the docks, and has been studied by scholars from different perspectives; see for example $[4,5,6,7,8,9]$ and the references therein.

\footnotetext{
${ }^{*}$ Corresponding author.

E-mail addresses: John-Graef@utc.edu (J.R. Graef), hos@ @rowan.edu (S. Ho), Lingju-Kong@utc.edu (L. Kong), min.wang @ kennesaw.edu (M. Wang).

Received December 10, 2018; Accepted June 1, 2019.
}

(C)2019 Journal of Nonlinear Functional Analysis 


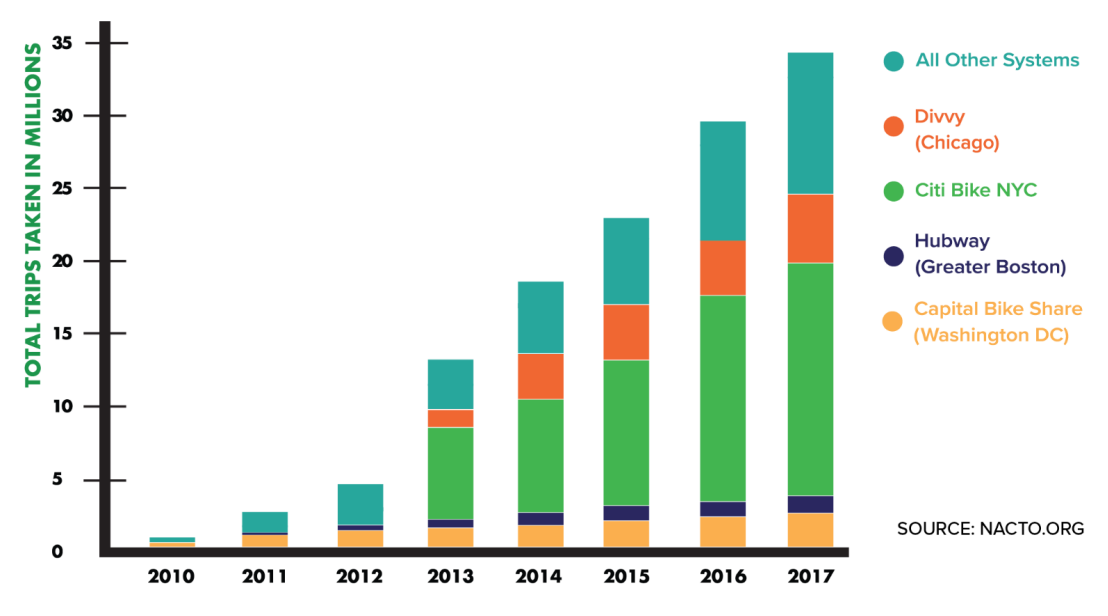

FIGURE 1. Growing ridership in USA [3]

As a classic modelling approach to study the dynamical evolutions of systems, differential equations (DEs) have been employed to investigate the bike share problem. But most existing DE bike share models are based on the mean-field method (see, for example, [1, 10, 11, 12]). Due to some technical reasons, the DE models based on the mean-field method do not directly consider the station inventory, i.e., the number of bikes at every station. Instead, they consider the proportions of stations with $k$ bikes at time $t$ (see [1]). This limitation motivates us to explore new inventory models that directly leverage the real data.

An exploratory data analysis (EDA) has been carried out on the Philadelphia Indego Bike Share System [13] in a preliminary project. Our analysis result reveals that the average waiting time of a bike at a station, i.e., the time a bike stays at a station until it is rented by the next user, follows the power-law tailed waiting time distributions, see Fig. 2. This pattern is further confirmed by the EDA on Atlanta Relay Bike Share System [14], see Fig. 3. In the DE study, power-law tailed distributions often lead to fractional terms. However, to the best of our knowledge, fractional order models have not been considered to investigate the bike share problems. Motivated by this finding, we will model the bike share system by fractional DEs (FDEs). In particular, we will consider the equation involving the $\alpha$-th left Riemann-Liouville fractional derivative of $u$ defined by

$$
\left(D_{0+}^{\alpha} u\right)(t)=\frac{1}{\Gamma(1-\alpha)} \frac{d}{d t} \int_{0}^{t}(t-s)^{-\alpha} u(s) d s, 0 \leq \alpha<1,
$$

provided the right-hand side exists, where $\Gamma(\cdot)$ is the Gamma function.

Fractional calculus is a subject with a long history and has been employed to model phenomenons from various areas. The resulting fractional order models have demonstrated superior performance in describing the long term memory and/or long range interaction compared to integer order models based on classical calculus. The reader is referred to [15, 16, 17, 18, 19, 20, 21, 22, 23, 24, 25] for the history and development of fractional calculus as well as some applications of fractional calculus in biology, image processing, mathematical finance, and physics.

Among the works cited above, many fractional models were obtained by replacing the integer order derivative(s) in classic models with fractional derivatives. The resulting models by this strategy are often 


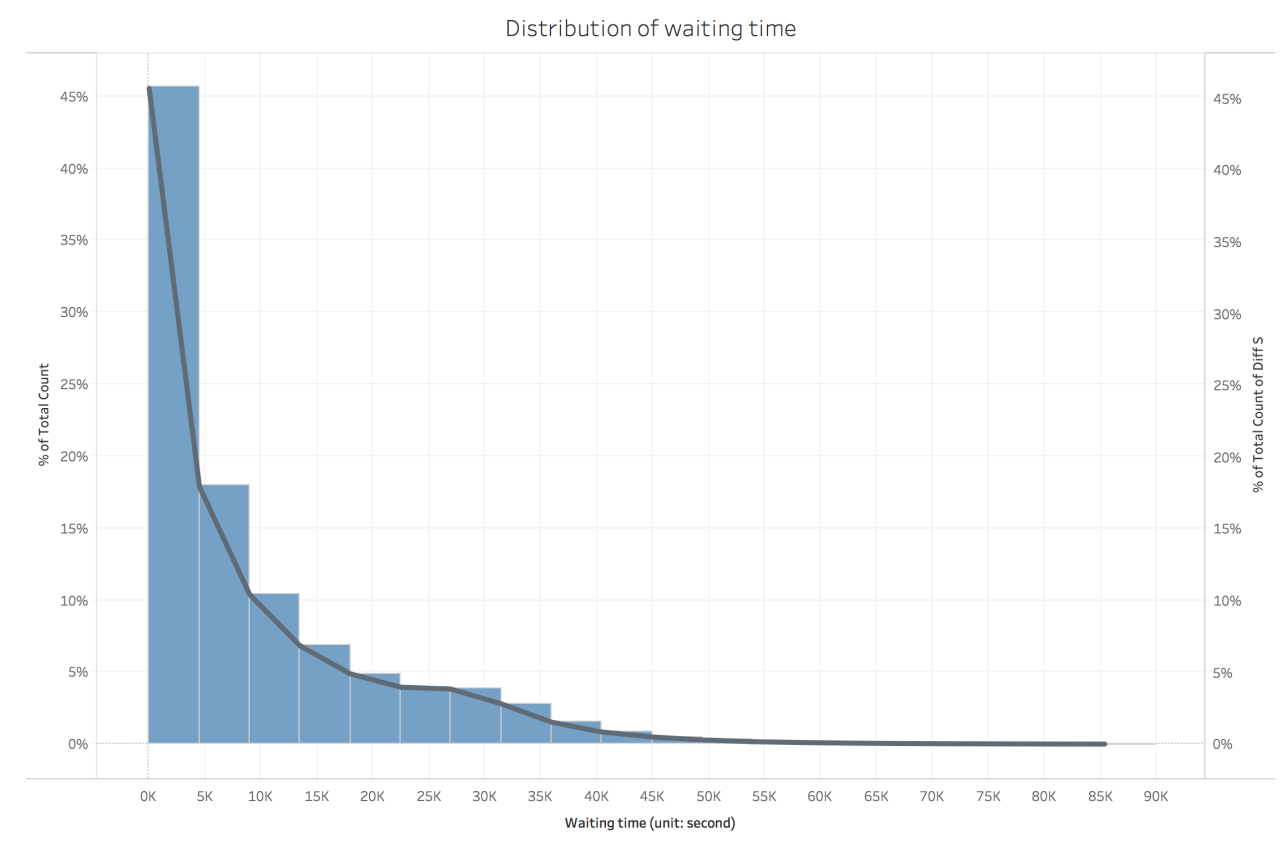

FIGURE 2. Average waiting time distribution of Indego System in Philadelphia based on data from Apr. 2015 - Jun. 2018.

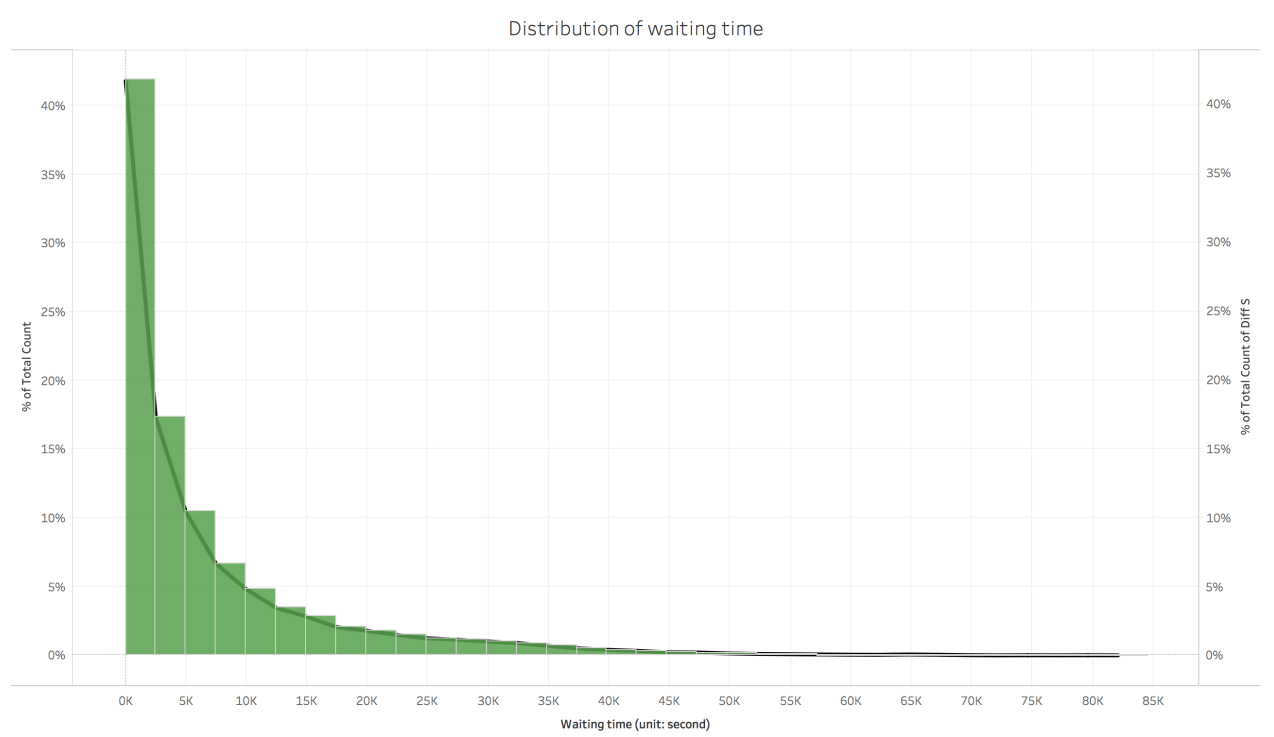

FIGURE 3. Average waiting time distribution of Relay System in Atlanta based on data from Sep. 2017 - Jul. 2018.

referred as the ad hoc fractional models [15, 16, 22]. As the authors of [22] point out, those ad hoc models may be mathematically interesting and have superior performance by offering more degrees of freedom to the models through the new fractional order(s), the modelling approach may not match the underlying physical process and may be difficult to interpret. The lack of interpretability has become a major obstacle of industrial applications of fractional models, especially in businesses with strict regulations such as financial institutions and credit bureaus. 
One feasible approach to derive interpretable fractional models is to focus on the underlying physical process and describe the long term memory or long range interactions by integral terms with appropriate kernels. The fractional terms will then be derived by mathematical transforms under certain assumptions. This approach has been used in $[15,16,20,22]$ to develop fractional compartment models. In this paper, we will employ a strategy similar to [22] to develop an interpretable fractional model for bike share systems.

The paper is organized as follows: After this introduction, the model will be developed in Section 2. Section 3 focuses on the analytic solution of the model. In Section 4 , the controllability of the model is investigated. Numerical simulations are also given there. Section 5 contains the conclusion and discussion.

\section{Fractional BiKe ShaRe MODEL}

In this section, we use FDE to model the inventory changes at bike share stations by considering the process to remove a bike from a station. Throughout this paper, the following assumptions are needed.

(H1) There are $N$ stations in total. The number of docks (maximum capacity) at Station $i$ is denoted by $U_{i}, i=1,2 \ldots, N$.

(H2) Let $u_{i}(t)$ be the number of bikes at the $i$-th station at time $t, t \geq 0$, and $\bar{u}_{i}=u_{i}(0), i=1,2, \ldots, N$.

(H3) The total arrival flux of Station $i$ at time $t$ is $q_{i}(t), i=1,2, \ldots, N$. For any small increment of time $\delta t$, the total number of bikes returned to Station $i$ between time $t$ and $t+\delta t$ is $q_{i}(t) \delta t$.

We assume that a bike in a station may be removed by either a Markovian removal process or a non-Markovian process.

(a) For $i=1, \ldots, N$, let $\omega_{i}(t)+o(\delta t)$ be the probability a bike will be removed by a Markovian removal process in the time interval $[t, t+\delta t]$ at Station $i$. By an argument similar to the one used in [26, Example 1.4], it is clear that the probability that no bike is removed by the Markovian process from $t_{0}$ to $t, \Theta_{i}\left(t, t_{0}\right)$, is defined by

$$
\Theta_{i}\left(t, t_{0}\right)=\exp \left(-\int_{t_{0}}^{t} \omega_{i}(s) d s\right) .
$$

It is easy to see that

$$
\frac{d}{d t} \Theta_{i}\left(t, t_{0}\right)=-\omega_{i}(t) \Theta_{i}\left(t, t_{0}\right), t \geq t_{0} \geq 0, i=1, \ldots, N .
$$

(b) For $i=1, \ldots, N$, let $\phi_{i}$ be the waiting time density function defined on $[0, \infty)$. So $\int_{0}^{t} \phi_{i}(\theta) d \theta$ is the probability that a bike is removed by a non-Markovian process by time $t$ and $\Phi_{i}$ defined by

$$
\Phi_{i}\left(t-t_{0}\right)=1-\int_{0}^{t-t_{0}} \phi_{i}(\theta) d \theta
$$

is the survival probability of a bike at Station $i$ from $t_{0}$ to $t$ due to the non-Markovian process. It is easy to see that $\Phi_{i}$ satisfies

$$
\frac{d}{d t} \Phi_{i}\left(t-t_{0}\right)=-\phi_{i}\left(t-t_{0}\right), t \geq t_{0}, i=1,2, \ldots, N .
$$

Then we have the following assumption by combining these two processes. 
(H4) A bike remains in a station until it is removed. The survival probability of a bike between $t_{0}$ and $t$, i.e. the probability that a bike entered a station at time $t_{0}$ and is still not removed at time $t$, is assumed to be a function of the time $t$ as well as the length of staying time $t-t_{0}$. We further assume that a bike cannot be returned and removed at the same time. So the survival probability is 1 when $t=t_{0}$. For $i=1,2, \ldots, N$, let the survival fuction of bikes at Station $i$ be $\Theta_{i}\left(t, t_{0}\right) \Phi_{i}\left(t-t_{0}\right)$ with $\Theta_{i}$ and $\Phi_{i}\left(t-t_{0}\right)$ defined by (2.1) and (2.3).

Remark 2.1. For $i=1, \ldots, N$, the survival function $\Theta_{i}\left(t, t_{0}\right) \Phi_{i}\left(t-t_{0}\right)$ represents the joint effort of a Markovian process and a non-Markovian process. In other words, the survival probability relies on not only the status at time $t$, but also the history between $t_{0}$ and $t$. This assumption offers more degrees of freedom to fit the distribution of waiting time based on real data.

From (H2)-(H4), for $t \geq 0$ and $i=1,2, \ldots, N$, we have

$$
u_{i}(t)=\bar{u}_{i} \Theta_{i}(t, 0) \Phi_{i}(t)+\int_{0}^{t} \Theta_{i}(t, \theta) \Phi_{i}(t-\theta) q_{i}(\theta) d \theta
$$

Then by (2.2), (2.4), and (2.5),

$$
\begin{aligned}
u_{i}^{\prime}(t)= & -\bar{u}_{i} \omega_{i}(t) \Theta_{i}(t, 0) \Phi_{i}(t)-\bar{u}_{i} \Theta_{i}(t, 0) \phi_{i}(t) \\
& +\Theta_{i}(t, t) \Phi_{i}(t-t) q_{i}(t)-\int_{0}^{t} \omega_{i}(t) \Theta_{i}(t, \theta) \Phi_{i}(t-\theta) q_{i}(\theta) d \theta \\
& -\int_{0}^{t} \Theta_{i}(t, \theta) \phi_{i}(t-\theta) q_{i}(\theta) d \theta \\
= & -\bar{u}_{i} \Theta_{i}(t, 0) \phi_{i}(t)+q_{i}(t)-\omega_{i}(t) u_{i}(t) \\
& -\int_{0}^{t} \Theta_{i}(t, \theta) \phi_{i}(t-\theta) q_{i}(\theta) d \theta,
\end{aligned}
$$

and from (2.5), we have

$$
\int_{0}^{t} \Theta_{i}(t, \theta) \Phi_{i}(t-\theta) q_{i}(\theta) d \theta=u_{i}(t)-\bar{u}_{i} \Theta_{i}(t, 0) \Phi_{i}(t) .
$$

Using (2.1), we have

$$
\frac{1}{\Theta_{i}(t, 0)}=\exp \left(\int_{0}^{t} \omega_{i}(s) d s\right)
$$

Thus, we have

$$
\int_{0}^{t} \Phi_{i}(t-\theta) \frac{q_{i}(\theta)}{\Theta_{i}(\theta, 0)} d \theta=\frac{u_{i}(t)}{\Theta_{i}(t, 0)}-\bar{u}_{i} \Phi_{i}(t) .
$$

Taking the Laplace transform on both sides of (2.7), we have

or

$$
\mathscr{L}\left\{\Phi_{i}\right\} \mathscr{L}\left\{\frac{q_{i}}{\Theta_{i}}\right\}=\mathscr{L}\left\{\frac{u_{i}}{\Theta_{i}}-\bar{u}_{i} \Phi_{i}\right\},
$$

$$
\begin{aligned}
\mathscr{L}\left\{\phi_{i}\right\} \mathscr{L}\left\{\frac{q_{i}}{\Theta_{i}}\right\} & =\frac{\mathscr{L}\left\{\phi_{i}\right\} \mathscr{L}\left\{\frac{u_{i}}{\Theta_{i}}-\bar{u}_{i} \Phi_{i}\right\}}{\mathscr{L}\left\{\Phi_{i}\right\}} \\
& =\frac{\mathscr{L}\left\{\phi_{i}\right\}}{\mathscr{L}\left\{\Phi_{i}\right\}} \mathscr{L}\left\{\frac{u_{i}}{\Theta_{i}}\right\}-\bar{u}_{i} \mathscr{L}\left\{\phi_{i}\right\}
\end{aligned}
$$


Therefore,

$$
\begin{aligned}
\int_{0}^{t} \phi_{i}(t-\theta) \frac{q_{i}(\theta)}{\Theta_{i}(\theta, 0)} d \theta & =\mathscr{L}^{-1}\left\{\frac{\mathscr{L}\left\{\phi_{i}\right\}}{\mathscr{L}\left\{\Phi_{i}\right\}} \mathscr{L}\left\{\frac{u_{i}}{\Theta_{i}}\right\}-\bar{u}_{i} \mathscr{L}\left\{\phi_{i}\right\}\right\} \\
& =\int_{0}^{t} K_{i}(t-\theta) \frac{u_{i}(\theta)}{\Theta_{i}(\theta, 0)} d \theta-\bar{u}_{i} \phi_{i}(t)
\end{aligned}
$$

where

$$
K_{i}(t)=\mathscr{L}^{-1}\left\{\frac{\mathscr{L}\left\{\phi_{i}\right\}}{\mathscr{L}\left\{\Phi_{i}\right\}}\right\} .
$$

Multiplying both sides of (2.8) by $\Theta_{i}(t, 0)$, we have

$$
\begin{aligned}
\int_{0}^{t} \Theta_{i}(t, \theta) \phi_{i}(t-\theta) q_{i}(\theta) d \theta= & \Theta_{i}(t, 0) \int_{0}^{t} K_{i}(t-\theta) \frac{u_{i}(\theta)}{\Theta_{i}(\theta, 0)} d \theta \\
& -\bar{u}_{i} \Theta_{i}(t, 0) \phi_{i}(t) .
\end{aligned}
$$

Hence, from (2.6) and (2.10), for any $t \geq 0$ and $i=1,2, \ldots, N$,

$$
u_{i}^{\prime}(t)=q_{i}(t)-\omega_{i}(t) u_{i}(t)-\Theta_{i}(t, 0) \int_{0}^{t} K_{i}(t-\theta) \frac{u_{i}(\theta)}{\Theta_{i}(\theta, 0)} d \theta .
$$

Note that (2.11) holds for any $K_{i}$ defined by (2.9) with $\Phi_{i}$ and $\phi_{i}$ satisfying (2.3). Next, we will derive the fractional model from (2.11) using an idea similar to that used in $[15,16,22]$. The following assumption on waiting time distribution for the non-Markovian process is needed.

(H5) For $i=1, \ldots, N$, the survival function $\Phi_{i}: \mathbb{R}^{+} \rightarrow \mathbb{R}^{+}$is assumed to be

$$
\Phi_{i}(t)=E_{\alpha_{i}}\left(-\left(\frac{t}{a_{i}}\right)^{\alpha_{i}}\right), \quad 0<\alpha_{i} \leq 1,
$$

where $a_{i}>0$ is a time scale parameter and $E_{\alpha}$ is the Mittag-Leffler function defined by

$$
E_{\alpha}(t)=\sum_{n=0}^{\infty} \frac{t^{n}}{\Gamma(n \alpha+1)} .
$$

Remark 2.2. By [24], $\Phi_{i}$ defined by (2.12) has a power-law asymptotic decay as $t \rightarrow \infty$, i.e., $\Phi_{i}(t) \sim t^{-\alpha_{i}}$ as $t \rightarrow \infty$. Then by (2.4), $\phi_{i} \sim t^{-1-\alpha_{i}}$ as $t \rightarrow \infty$. This is consistent with our EDA results in Fig. 2 and 3.

Taking the Laplace transform of (2.12) gives

$$
\mathscr{L}\left\{\Phi_{i}\right\}=\frac{1}{s\left(1+\left(a_{i} s\right)^{\left.-\alpha_{i}\right)}\right.} .
$$

By (2.3) and (2.4),

$$
\mathscr{L}\left\{\phi_{i}\right\}=1-s \mathscr{L}\left\{\Phi_{i}\right\} .
$$

Hence by (2.9),

$$
\mathscr{L}\left\{K_{i}\right\}=a_{i}^{-\alpha_{i}} s^{1-\alpha_{i}} .
$$

Therefore,

$$
\begin{aligned}
\int_{0}^{t} K_{i}(t-\theta) \frac{u_{i}(\theta)}{\Theta_{i}(\theta, 0)} d \theta & =\mathscr{L}^{-1}\left\{\mathscr{L}\left\{K_{i}\right\} \mathscr{L}\left\{\frac{u_{i}}{\Theta_{i}}\right\}\right\} \\
& =\mathscr{L}^{-1}\left\{a_{i}^{-\alpha_{i}} s^{1-\alpha_{i}} \mathscr{L}\left\{\frac{u_{i}}{\Theta_{i}}\right\}\right\} .
\end{aligned}
$$


Note that

$$
\begin{aligned}
& \mathscr{L}\left\{D_{0+}^{1-\alpha_{i}}\left(\frac{u_{i}}{\Theta_{i}}\right)\right\}=\mathscr{L}\left\{\frac{d}{d t}\left(\frac{1}{\Gamma\left(\alpha_{i}\right)} \int_{0}^{t}(t-\theta)^{\alpha_{i}-1} \frac{u_{i}(\theta)}{\Theta_{i}(\theta, 0)} d \theta\right)\right\} \\
= & s^{1-\alpha_{i}} \mathscr{L}\left\{\frac{u_{i}}{\Theta_{i}}\right\}-\left.\left(\frac{1}{\Gamma\left(\alpha_{i}\right)} \int_{0}^{t}(t-\theta)^{\alpha_{i}-1} \frac{u_{i}(\theta)}{\Theta_{i}(\theta, 0)} d \theta\right)\right|_{t=0} \\
= & s^{1-\alpha_{i}} \mathscr{L}\left\{\frac{u_{i}}{\Theta_{i}}\right\},
\end{aligned}
$$

where $D_{0+}^{1-\alpha_{i}}\left(\frac{u_{i}}{\Theta_{i}}\right)$ is the $\left(1-\alpha_{i}\right)$-th left Riemann-Liouville fractional derivative of $\frac{u_{i}}{\Theta_{i}}$ defined by (1.1). Hence (2.14) and (2.15) imply

$$
\int_{0}^{t} K_{i}(t-\theta) \frac{u_{i}(\theta)}{\Theta_{i}(\theta, 0)} d \theta=a_{i}^{-\alpha_{i}} D_{0+}^{1-\alpha_{i}}\left(\frac{u_{i}(t)}{\Theta_{i}(t, 0)}\right) .
$$

Therefore by $(\mathrm{H} 1),(\mathrm{H} 2),(2.11)$, and (2.16), the FDE model of the status of the bike stations is given by

$$
\left\{\begin{array}{l}
u_{i}^{\prime}=q_{i}(t)-\omega_{i}(t) u_{i}-\Theta_{i}(t) a_{i}^{-\alpha_{i}} D_{0+}^{1-\alpha_{i}}\left(\frac{u_{i}}{\Theta_{i}}\right), \\
u_{i}(0)=\bar{u}_{i}, t>0, i=1, \ldots, N
\end{array}\right.
$$

with

$$
\Theta_{i}(t)=\Theta_{i}(t, 0)=\exp \left(-\int_{0}^{t} \omega(s) d s\right) .
$$

Remark 2.3. (a) It is easy to see that

$$
\omega_{i}(t) u_{i}(t)+\Theta_{i}(t) a_{i}^{-\alpha_{i}} D_{0+}^{1-\alpha_{i}}\left(\frac{u_{i}}{\Theta_{i}}\right)
$$

is the outgoing flux of Station $i, i=1,2, \ldots, N$.

(b) The arrival flux $q_{i}, i=1, \ldots, N$, may be determined by fitting the historical data. We will assume $q_{i}$ is known in Section 3.

\section{AnAlytic SOlutions of The FDE MOdEL}

In this section, we will investigate the solutions of Eq. (2.17). The following lemma will be the foundation of our conclusion.

Lemma 3.1. Assume $h \in C\left(\mathbb{R}_{+}, \mathbb{R}\right)$. The initial value problem (IVP)

$$
\left\{\begin{array}{l}
X^{\prime}+a D_{0+}^{1-\alpha} X=h(t), t>0 \\
X(0)=\tilde{X}
\end{array}\right.
$$

has a unique solution given by

$$
X(t)=\int_{0}^{t} E_{\alpha}\left(-a(t-s)^{\alpha}\right) h(s) d s+\tilde{X} E_{\alpha}\left(-a t^{\alpha}\right) .
$$

Proof. By taking the Laplace transform of (3.1), we have

$$
s \mathscr{L}\{X\}-X(0)+a s^{1-\alpha} \mathscr{L}\{X\}=\mathscr{L}\{h\},
$$

or

$$
\mathscr{L}\{X\}=\frac{s^{\alpha-1}}{s^{\alpha}+a}(\mathscr{L}\{h\}+X(0)) .
$$


Note that

$$
\mathscr{L}\left\{E_{\alpha}\left(-a t^{\alpha}\right)\right\}=\frac{s^{\alpha-1}}{s^{\alpha}+a} .
$$

Then we have

$$
X(t)=\int_{0}^{t} E_{\alpha}\left(-a(t-s)^{\alpha}\right) h(s) d s+X(0) E_{\alpha}\left(-a t^{\alpha}\right), t>0 .
$$

The uniqueness follows immediately.

Remark 3.2. By (3.1) and (3.2), it is easy to see that $E_{\alpha}\left(-a t^{\alpha}\right)$ is a solution of

$$
\left\{\begin{array}{l}
X^{\prime}+a D_{0+}^{1-\alpha} X=0, t>0 \\
X(0)=1
\end{array}\right.
$$

We are now ready to study Eq. (2.17).

Theorem 3.3. Eq. (2.17) has a unique solution given by

$$
\begin{aligned}
u_{i}(t)= & \int_{0}^{t} E_{\alpha_{i}}\left(-\left(\frac{t-s}{a_{i}}\right)^{\alpha_{i}}\right) \Theta_{i}(t, s) q_{i}(s) d s+\bar{u}_{i} \Theta_{i}(t, 0) E_{\alpha_{i}}\left(-\left(\frac{t}{a_{i}}\right)^{\alpha_{i}}\right), \\
& t>0, i=1, \ldots, N .
\end{aligned}
$$

Proof. Let

$$
v_{i}(t)=\frac{u_{i}(t)}{\Theta_{i}(t)}=\exp \left(\int_{0}^{t} \omega_{i}(s) d s\right) u_{i}(t)
$$

with $\Theta_{i}$ defined by (2.18). Then

$$
v_{i}^{\prime}=\omega_{i}(t) \exp \left(\int_{0}^{t} \omega_{i}(s) d s\right) u_{i}(t)+\exp \left(\int_{0}^{t} \omega_{i}(s) d s\right) u_{i}^{\prime} .
$$

It is easy to verify that (2.17) is equivalent to

$$
\left\{\begin{array}{l}
v_{i}^{\prime}=Q_{i}(t)-a_{i}^{-\alpha_{i}} D_{0+}^{1-\alpha_{i}} v_{i}, \quad t>0, i=1, \ldots, N, \\
v_{i}(0)=\bar{u}_{i}
\end{array}\right.
$$

with

$$
Q_{i}(t)=\exp \left(\int_{0}^{t} \omega_{i}(s) d s\right) q_{i}(t)
$$

By Lemma 3.1, Eq. (3.5) has the unique solution

$$
v_{i}(t)=\int_{0}^{t} E_{\alpha_{i}}\left(-\left(\frac{t-s}{a_{i}}\right)^{\alpha_{i}}\right) Q_{i}(s) d s+\bar{u}_{i} E_{\alpha_{i}}\left(-\left(\frac{t}{a_{i}}\right)^{\alpha_{i}}\right) .
$$

Then (3.4) follows immediately. 


\section{Controllability of the FDE model}

By Theorem 3.3, the solution of Eq. (2.17) replies on the arrival flux $q_{i}, i=1, \ldots, N$. This is consistent with the intuitive understanding of the problem. Moreover, this result suggests that we investigate the feasibility to control the inventory at each station by controlling the arrival flux.

In the sequel, we will view $q_{i}$ as a control and let

$$
u_{i}^{\bar{u}_{i}, q_{i}}(t), i=1, \ldots, N
$$

denote the solution of Eq. (2.17) with initial conditions $\left(\bar{u}_{1}, \ldots, \bar{u}_{N}\right) \in \mathbb{R}^{N}$ and control $\left(q_{1}, \ldots, q_{N}\right) \in$ $C\left(\mathbb{R}_{+}, \mathbb{R}^{N}\right)$. The following definitions are taken from [27].

Definition 4.1. (a) We say that $\left(q_{1}, \ldots, q_{N}\right)$ transfers $\overline{\mathbf{u}}=\left(\bar{u}_{1}, \ldots, \bar{u}_{N}\right) \in \mathbb{R}^{N}$ to $\hat{\mathbf{u}}=\left(\hat{u}_{1}, \ldots, \hat{u}_{N}\right) \in \mathbb{R}^{N}$ at $T>0$ if

$$
u_{i}^{\bar{u}_{i}, q_{i}}(T)=\hat{u}_{i}, i=1, \ldots, N .
$$

We then also say that $\hat{\mathbf{u}}$ is reachable from $\overline{\mathbf{u}}$ at $T$. The set $\mathscr{A}(T ; \overline{\mathbf{u}}) \subset \mathbb{R}^{N}$ defined by

$$
\mathscr{A}(T ; \overline{\mathbf{u}})=\left\{\hat{\mathbf{u}} \mid \text { There exists a control }\left(q_{1}, \ldots, q_{N}\right) \text { that transfers } \overline{\mathbf{u}} \text { to } \hat{\mathbf{u}} \text { at } T\right\}
$$

is the reachable set at $T$ from $\overline{\mathbf{u}}$.

(b) Eq. (2.17) is said to be controllable if for arbitrary $\left(\bar{u}_{1}, \ldots, \bar{u}_{N}\right),\left(\hat{u}_{1}, \ldots, \hat{u}_{N}\right) \in \mathbb{R}^{N}$, there exists a control $\left(q_{1}, \ldots, q_{N}\right) \in C\left(\mathbb{R}_{+}, \mathbb{R}^{N}\right)$ and some $T>0$ such that $\left(q_{1}, \ldots, q_{N}\right)$ transfers $\left(\bar{u}_{1}, \ldots, \bar{u}_{N}\right)$ to $\left(\hat{u}_{1}, \ldots, \hat{u}_{N}\right)$ at $T$.

We have the following result on the controllability of Eq. (2.17).

Theorem 4.2. (a) For arbitrary $\left(\bar{u}_{1}, \ldots, \bar{u}_{N}\right),\left(\hat{u}_{1}, \ldots, \hat{u}_{N}\right) \in \mathbb{R}^{N}$ and $T>0$, let $\left(\hat{q}_{1}, \ldots, \hat{q}_{N}\right)$ be defined by

$$
\hat{q}_{i}(t)=\frac{\hat{u}_{i}-\bar{u}_{i} \Theta_{i}(T, 0) E_{\alpha_{i}}\left(-\left(\frac{T}{a_{i}}\right)^{\alpha_{i}}\right)}{\int_{0}^{T}\left[E_{\alpha_{i}}\left(-\left(\frac{T-s}{a_{i}}\right) \alpha_{i}\right) \Theta_{i}(T, s)\right]^{2} d s} E_{\alpha_{i}}\left(-\left(\frac{T-t}{a_{i}}\right)^{\alpha_{i}}\right) \Theta_{i}(T, t),
$$

$i=1, \ldots, N$. Then $\left(\hat{q}_{1}, \ldots, \hat{q}_{N}\right)$ transfers $\left(\bar{u}_{1}, \ldots, \bar{u}_{N}\right)$ to $\left(\hat{u}_{1}, \ldots, \hat{u}_{N}\right)$ at $T$, and so Eq. (2.17) is controllable.

(b) Among all the controls $\left(q_{1}, \ldots, q_{N}\right)$ transferring $\left(\bar{u}_{1}, \ldots, \bar{u}_{N}\right)$ to $\left(\hat{u}_{1}, \ldots, \hat{u}_{N}\right)$ at $T,\left(\hat{q}_{1}, \ldots, \hat{q}_{N}\right)$ minimizes the quantity

$$
\sum_{i=1}^{N} \int_{0}^{T} q_{i}^{2}(s) d s
$$

Proof. Part (a) may be verified by direct computation. We omit the details.

Part (b). By (3.4), for any control $\left(q_{1}, \ldots, q_{N}\right)$ transferring $\left(\bar{u}_{1}, \ldots, \bar{u}_{N}\right)$ to $\left(\hat{u}_{1}, \ldots, \hat{u}_{N}\right)$ at $T$,

$$
\hat{u}_{i}-\bar{u}_{i} \Theta_{i}(T, 0) E_{\alpha_{i}, 1}\left(-\left(\frac{T}{a_{i}}\right)^{\alpha_{i}}\right)=\int_{0}^{T} E_{\alpha_{i}, 1}\left(-\left(\frac{T-s}{a_{i}}\right)^{\alpha_{i}}\right) \Theta_{i}(T, s) q_{i}(s) d s,
$$


$i=1, \ldots, N$. Note that by (4.1) and (4.2),

$$
\begin{aligned}
\int_{0}^{T} q_{i}(s) \hat{q}_{i}(s) d s= & \frac{\left[\hat{u}_{i}-\bar{u}_{i} \Theta_{i}(T, 0) E_{\alpha_{i}}\left(-\left(\frac{T}{a_{i}}\right) \alpha^{\alpha_{i}}\right)\right] \int_{0}^{T} q_{i}(s) E_{\alpha_{i}}\left(-\left(\frac{T-s}{a_{i}}\right)^{\alpha_{i}}\right) \Theta_{i}(T, s) d s}{\int_{0}^{T}\left[E_{\alpha_{i}}\left(-\left(\frac{T-s}{a_{i}}\right)^{\alpha_{i}}\right) \Theta_{i}(T, s)\right]^{2} d s} \\
& =\frac{\left[\hat{u}_{i}-\bar{u}_{i} \Theta_{i}(T, 0) E_{\alpha_{i}}\left(-\left(\frac{T}{a_{i}}\right)^{\alpha_{i}}\right)\right]^{2}}{\int_{0}^{T}\left[E_{\alpha_{i}}\left(-\left(\frac{T-s}{a_{i}}\right) \alpha_{i}\right) \Theta_{i}(T, s)\right]^{2} d s}=\int_{0}^{T}\left[\hat{q}_{i}(s)\right]^{2} d s .
\end{aligned}
$$

Therefore,

$$
\begin{aligned}
\int_{0}^{T}\left[q_{i}(s)\right]^{2} d s= & \int_{0}^{T}\left[q_{i}(s)-\hat{q}_{i}(s)\right]^{2} d s+\int_{0}^{T}\left[\hat{q}_{i}(s)\right]^{2} d s \\
& +2 \int_{0}^{T}\left[q_{i}(s)-\hat{q}_{i}(s)\right] \hat{q}_{i}(s) d s \\
= & \int_{0}^{t}\left[q_{i}(s)-\hat{q}_{i}(s)\right]^{2} d s+\int_{0}^{t}\left[\hat{q}_{i}(s)\right]^{2} d s
\end{aligned}
$$

by (4.3). Hence, $\left(\hat{q}_{1}, \ldots, \hat{q}_{N}\right)$ minimizes the integral

$$
\sum_{i=1}^{N} \int_{0}^{T} q_{i}^{2}(s) d s
$$

Remark 4.3. (a) In practice, the arrival flux will always be nonnegative. Therefore, by (3.4), for any $T>0$ and $i=1, \ldots, N$,

$$
u_{i}(T) \geq \bar{u}_{i} \Theta_{i}(T, 0) E_{\alpha_{i}}\left(-\left(\frac{T}{a_{i}}\right)^{\alpha_{i}}\right) .
$$

In addition, by (H1), the maximum capacity at Station i is $U_{i}$. So a reasonably reachable set at $T$ of (2.17) is

$$
\mathscr{A}(T ; \overline{\mathbf{u}})=\Pi_{i=1}^{N}\left[\bar{u}_{i} \Theta_{i}(T, 0) E_{\alpha_{i}}\left(-\left(\frac{T}{a_{i}}\right)^{\alpha_{i}}\right), U_{i}\right] .
$$

(b) Theorem 4.2 shows that it is feasible to reach the expected inventory at a bike station at time $T$ by controlling the arrival flux. This result may contribute to the development of new inventory rebalancing strategies.

Next, we use numerical simulations to demonstrate the applicability of Theorem 4.2. The values of the parameters in (2.17) and the initial/terminal values in the following example are chosen for illustrative purposes and are not from actual data.

Example 4.4. Consider a bike share system with two stations, i.e., $N=2$. Assume that $\alpha_{1}=0.3$, $\alpha_{2}=0.5, a_{1}=0.4, a_{2}=0.3, T=30$ minutes, the initial values are $\bar{u}_{1}=6$ and $\bar{u}_{2}=2$, and the terminal values are $\hat{u}_{1}=10$ and $\hat{u}_{2}=15$. The removal probabilities $\omega_{1}$ and $\omega_{2}$ are taken to be two polynomials of degree 5 with the coefficients given in Table 1 and graphs given in Fig. 4.

By Theorem 4.2, the controls $\hat{q}_{1}$ and $\hat{q}_{2}$ defined by (4.1) will transfer $\left(\bar{u}_{1}, \bar{u}_{2}\right)$ to $\left(\hat{u}_{1}, \hat{u}_{2}\right)$ at $T$. The graphs of $\hat{q}_{i}, i=1,2$, are given in Fig. 5. The solution of Eq. (2.17) is then computed by (3.4). The graphs and the phase portrait of the solution are given in Fig. 6 and 7 respectively. It is clear that the simulation results are consistent with our conclusions. 
FRACTIONAL BIKE SHARE MODEL

TABLE 1. The parameters for Eq. (2.17).

\begin{tabular}{|c||ccc|}
\hline \hline function & $a_{5}$ & $a_{4}$ & $a_{3}$ \\
\hline$\omega_{1}$ & 0 & 0.000000000000877 & -0.000000002713261 \\
\hline$\omega_{2}$ & 0.000000000000006 & -0.000000000021770 & 0.000000027091416 \\
\hline \hline function & $a_{2}$ & $a_{1}$ & $a_{0}$ \\
\hline$\omega_{1}$ & 0.000003846127552 & -0.001178086841168 & 0.138020635158977 \\
\hline$\omega_{2}$ & -0.000012680449458 & 0.002087155685997 & -0.044611654946751 \\
\hline
\end{tabular}

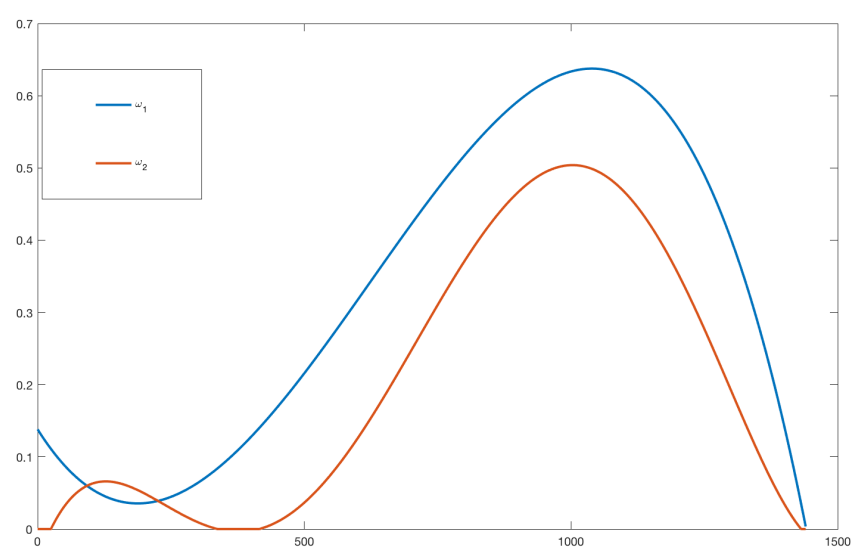

FIGURE 4. Graphs of $\omega_{1}$ and $\omega_{2}$.

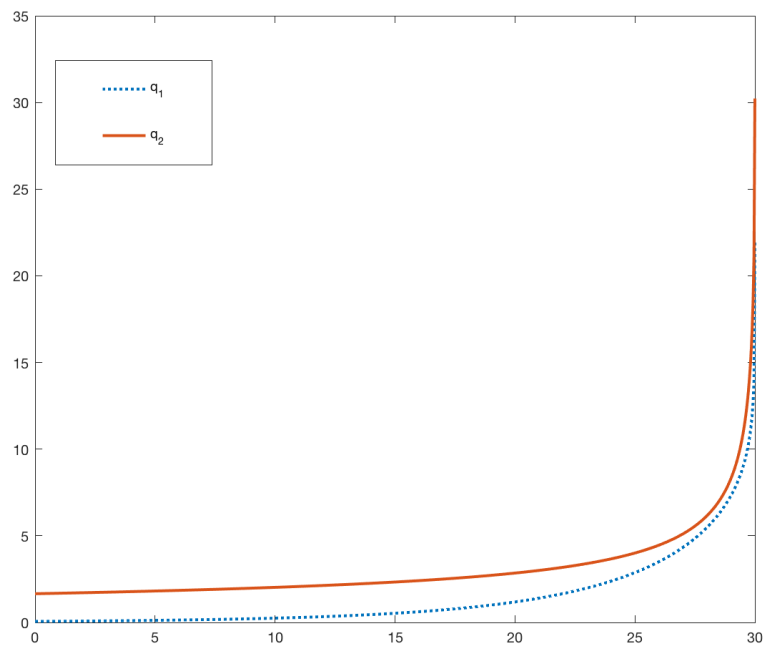

FIGURE 5. Graphs of $\hat{q}_{1}$ and $\hat{q}_{2}$. 


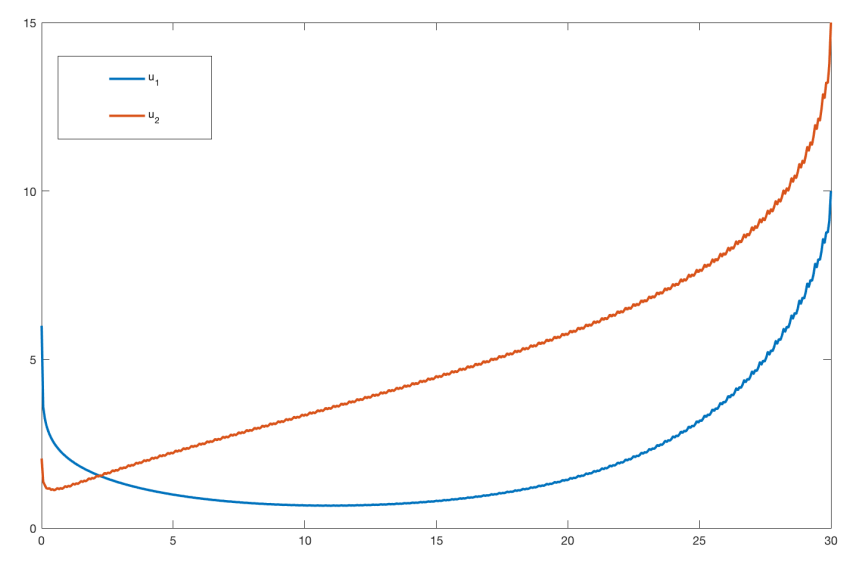

FIGURE 6. Solutions of Eq. (2.17) under the controls $\hat{q}_{1}$ and $\hat{q}_{2}$.

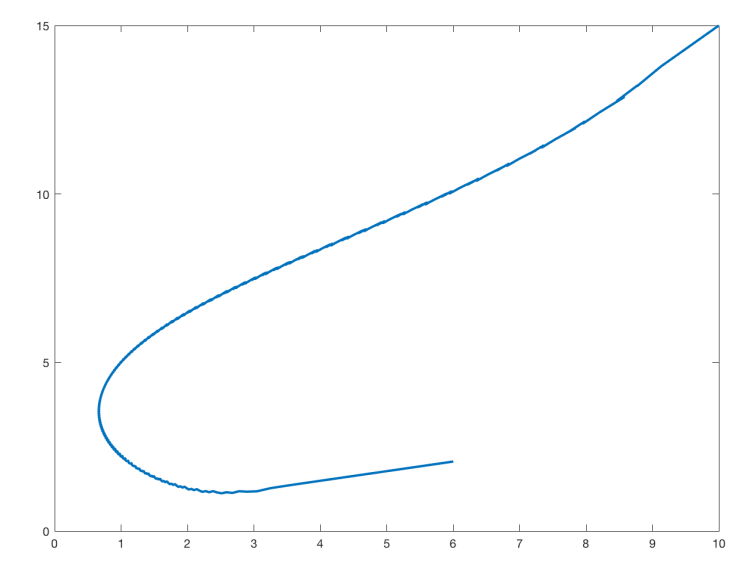

FIGURE 7. Trajectory of Eq. (2.17) under the controls $\hat{q}_{1}$ and $\hat{q}_{2}$.

\section{CONCLUSION AND DISCUSSION}

In this paper, we develop a linear fractional differential equation model to describe the inventory of bike share stations. Instead of the ad hoc approach, we derive the fractional term by investigating the underlying removal processes of a bike at a station. A closed form solution of the model is then obtained. We further consider a control problem based on our FDE model. Numerical simulation is used to verify our results. Comparing with traditional integer order DE models, our model offers more degrees of freedom without complicating the model. Our results show that it is feasible to control the inventory at each bike station by adjusting the arrival flux. This finding has potential applications in the development of new inventory rebalancing strategy.

\section{Funding}

J.R. Graef and L. Kong's research was supported in part by a University of Tennessee at Chattanooga SimCenter - Center of Excellence in Applied Computational Science and Engineering (CEACSE) grant. 
S. Ho and M. Wang's research in this paper is supported by the National Science Foundation under Grant No. 1830489.

\section{REFERENCES}

[1] S. Tao, J. Pender, A stochastic analysis of bike sharing systems, preprint, arXiv:1708.08052, 2017.

[2] NACTO Bike Share Initiative, Bike Share in the US: 2010-2016, Retrieved from https://nacto.org/bike-share-statistics$2016 /$.

[3] NACTO Bike Share Initiative, Bike Share in the U.S.: 2017, Retrieved from https://nacto.org/bike-share-statistics-2017/.

[4] D. Chemla, F. Meunier, R. Wolfler Calvo, Bike sharing systems: Solving the static rebalancing problem, Discrete Optim. 10 (2013), 120-146.

[5] C. Médard de Chardon, G. Caruso, I. Thomas, Bike-share rebalancing strategies, patterns, and purpose, J. Transport Geography 55 (2016), 22-39.

[6] J. Pfrommer, J. Warrington, G. Schildbach, M. Morari, Dynamic vehicle redistribution and online price incentives in shared mobility systems, IEEE Trans. Intelligent Transportation Systems 15 (2014), 1567-1578.

[7] T. Preisler, T. Dethlefs, W. Renz, Data-adaptive simulation: cooperativeness of users in bike-sharing systems, In: W. Kersten, T. Blecker, C.M. Ringle, (eds.) Proceedings of the Hamburg International Conference of Logistics, vol. 20. epubli GmbH (2015).

[8] T. Preisler, T. Dethlefs, W. Renz, Self-organizing redistribution of bicycles in a bike-sharing system based on decentralized control, Proc. Federated Conference on Computer Science and Information Systems 8 (2016), 1471-1480.

[9] T. Raviv, M. Tzur, I. A. Forma, Static repositioning in a bike-sharing system: models and solution approaches, EURO J. Trans. Logist. 2 (2013), 187-229.

[10] C. Fricker, N. Gast, Incentives and redistribution in homogeneous bike-sharing systems with stations of finite capacity, EURO J. Trans. Logist. 5 (2016), 261-291.

[11] C. Fricker, N. Gast, H. Mohamed, Mean field analysis for inhomogeneous bike sharing systems, Proceedings of the twenty-third international meeting on probabilistic, combinatorial, and asymptotic methods for the analysis of algorithms, DMTCS, pp. 365-376, (2012)

[12] Q. Li, C. Chen, R. Fan, L. Xu, J. Ma, Queueing analysis of a large-scale bike sharing system through mean-field theory, arXiv Preprint: arXiv:1603.09560, pp. 1C50, (2016).

[13] Anonymized Indego trip data, Retrieved from https://www.rideindego.com/about/data/ (accessed Aug. 19, 2018).

[14] Anonymized relay trip data, Retrieved from https://relaybikeshare.com/system-data/ (accessed Aug. 19, 2018).

[15] C. Angstmann, B. Henry, A. McGann, A fractional-order infectivity SIR model, Physica A: Statistical Mechanics and its Applications 452 (2016), 86-93.

[16] C. Angstmann, B. Henry, A. McGann, A fractional order recovery SIR model from a stochastic process, Bull. Math. Biology 78 (2016), 468-499.

[17] A.A. Kilbas, H.M. Srivastava, J.J. Trujillo, Theory and Applications of Fractional Differential Equations, North-Holland Mathematics Studies, vol. 204. Elsevier, Amsterdam, (2006).

[18] K.S. Miller, B. Ross, An Introduction to the Fractional Calculus and Fractional Differential Equations, Wiley-Interscience, Nerw York, (1993).

[19] I. Podlubny, Fractional Differential Equations, Academic Press, New York, (1998).

[20] V. Tarasov, Fractional Dynamics: Applications of Fractional Calculus to Dynamics of Particles, Fields and Media, Springer-Verlag, New York, (2011).

[21] Q. Yang, D. Chen, T. Zhao, Y. Chen, Fractional calculus in image processing: a review, Fractional Calculus Appl. Anal. 19 (2016), 1222-1249.

[22] C. Angstmann, A. Erickson, B. Henry, A. McGann, J. Murray, J. Nichols, Fractional order compartment models, SIAM J. Appl. Math. 77 (2017), 430-446.

[23] A.C.D. Faria, J. Veiga, A.J. Lopes, P.L. Melo, Forced oscillation, integer and fractional-order modeling in asthma, Computer Methods and Programs in Biomedicine 128 (2016), 12-26.

[24] F. Mainardi, On some properties of the Mittag-Leffler function $E_{\alpha}\left(-t^{\alpha}\right)$, completely monotone for $t>0$ with $0<\alpha<1$, Discrete \& Continuous Dynamical Systems-B 19 (2014), 2267-2278. 
[25] G. Jumarie, Derivation and solutions of some fractional Black-Scholes equations in coarse-grained space and time. Application to Merton's optimal portfolio, Comput. Math. Appl. 59 (2010), 1142-1164.

[26] E. Allen, Modeling with Itô Stochastic Differential Equations, Springer, New York, (2007).

[27] J. Zabczyk, Mathematical Control Theory: An Introduction, Birkhäuser, Boston, (1992). 